\title{
NOTE ON THE RATIO OF MASS TO WEIGHT FOR BISMUTH AND ALUMINUM.
}

By H. A. WiLson.

SYNOPSIS.

The ratio of mass to weight has been shown to be the same for bismuth and aluminum to within one in one million, by the method of Eötvös.

R. BRUSH ${ }^{1}$ has recently described some experiments on the gravitational attractions exerted by several substances and on the ratio of mass to weight for some of them. Contrary to the results of previous experimenters he finds this ratio different for different substances and the attractions per unit mass also different. Mr. Brush's experiments appear to have been conducted with some care and it is not easy to suggest sources of error which might account for his surprising results. In the case of his pendulum experiments he does not state that he corrected for the buoyancy of the air and this effect, if not allowed for, would be sufficient to produce an error of the same order as the differences he found.

Mr. Brush found the greatest difference in the attractions in the case of aluminum and bismuth, so I have made a comparison of the ratios of mass to weight for these two substances by the very excellent method of Eötvös. ${ }^{2}$ The result shows that this ratio is the same for these two substances to within one part in one million.

Two small cylinders, one of bismuth and one of aluminum, and each weighing about one gram were attached to the ends of a horizontal aluminum wire six cms. long which was soldered to a vertical wire at its middle point. The vertical wire was suspended by a fine platinum wire, thus forming a torsion balance. A mirror was carried by the vertical wire and the balance was suspended in a suitable metal case having a glass window. The case could be turned about a vertical axis on a stand provided with levelling screws. A telescope, provided with illuminated cross wires in its eye-piece was attached to the case and the apparatus could be adjusted so that the image of the cross wires formed by light reflected from the mirror could be made to coincide with another cross wire in the eye-piece.

I Phys. Rev. (2), XIX., p. I25, I92I.

2 Mathematische und Naturwissenschaftliche Berichte aus Ungarn, Bd. 8, S. 64, I89r. 
The case was turned so that the balance beam was approximately East and West and was then turned through two rightangles. It was found that after turning through two rightangles the image of the cross wires came to rest not more than one quarter of a millimeter from its previous position in the eye-piece. The lower end of the vertical wire of the balance dipped into a small vessel containing castor oil which gave sufficient damping. To prevent twisting of the torsion wire when the case was turned through two rightangles stops were provided which carried the beam round with the case. These stops were put in position after the telescope was adjusted so that the image coincided with the cross wires and were removed after the case had been rotated.

The time of one complete vibration of the beam was about three minutes.

The theory of the experiment is as follows. Owing to the rotation of the earth part of the attraction of the earth on a body is used up in keeping the body on its circular path.

If $r$ denotes the radius of the earth and $\omega$ its angular velocity, then in latitude $\theta$, the horizontal component of the centrifugal force on a body of mass $m$ is $m r \omega^{2} \cos \theta \sin \theta$. The attraction of the earth is $m g$ towards its center so that if $\phi$ denotes the inclination of the apparent weight to the earth's radius

$$
\phi=\frac{r \omega^{2} \cos \theta \sin \theta}{g} .
$$

For another body suppose

$$
\phi^{\prime}=\frac{r \omega^{2} \cos \theta \sin \theta}{g^{\prime}} .
$$

If the two bodies have nearly equal masses and are suspended East and West on a torsion balance, as in Eötvös' experiment, there will be a couple acting on the balance equal to $m g d\left(\phi-\phi^{\prime}\right)$ where $d$ is half the length of the balance.

If $T$ is the time of one oscillation of the balance $T=2 \pi d \sqrt{2 m / \mu}$, where $\mu$ is the torsion coefficient of the wire. Hence $\mu=8 \pi^{2} m d^{2} / T^{2}$. The couple $m g d\left(\phi^{\prime}-\phi\right)$ will twist the wire through an angle $\Delta$ given by

$$
\mu \Delta=m g\left(\phi^{\prime}-\phi\right) d .
$$

When the apparatus is turned through two rightangles the wire will be twisted through an angle $2 \Delta$ and

$$
2 \Delta=\frac{2 m g\left(\phi-\phi^{\prime}\right) d}{\mu}=\frac{\left(g-g^{\prime}\right) T^{2} \gamma w^{2} \cos \theta \sin \theta}{4 \pi^{2} g^{\prime}} .
$$


If $t$ denotes the time of one revolution of the earth then $\omega=2 \pi / t$ and

$$
\frac{g-g^{\prime}}{g^{\prime}}=(2 \Delta) \frac{2 d t^{2}}{r T^{2} \sin 2 \theta} .
$$

For Houston $\theta=30^{\circ}$ so with $T=180$ secs. $g=980$ and $d=3 \mathrm{cms}$. we get

$$
\frac{g-g^{\prime}}{g^{\prime}}=25 \times \mathrm{IO}^{-4} \times(2 \Delta) .
$$

The experiments showed that $2 \Delta$ was less than about $\mathrm{I} / 2500$, so that $\left(g-g^{\prime}\right) / g^{\prime}$ was less than about $1^{-6}$.

We conclude therefore that the acceleration of gravity is the same for bismuth and aluminum to within one part in one million.

Rice InSTITUTE,

Houston, Texas,

March 25, I922. 